\section{Modelling cost-effective therapies}

To the Editor: In the present era of rapidly increasing medical costs, there is a need for doctors to assist in controlling these costs, in addition to providing quality care to all patients. This requires inclusion of the parameter of 'value' in medical practice. Value has been defined as 'outcome divided by costs. ${ }^{[1]}$

In Fig. 1, a model of the changes in outcome with increasing costs is proposed. According to the model, the change follows a sigmoid curve pattern. This pattern is widely observed in biology, e.g. the dose-response effect to irradiation or chemotherapy in oncology, and is also seen in the diffusion of innovation in economics.

The model is semiquantitative and does not specify the endpoint for outcome on the vertical axis or the cost in numbers on the horizontal axis. The endpoint used for outcomes will vary depending on the disease being treated. For example, in oncology the endpoints may be overall survival or quality of life parameters. The cost will vary depending on the therapeutic options available.

As can be seen in the model, value is initially low (A - B in Fig. 1), then increases (B - C), and then decreases again (C - D).

In cost-effective practice, doctors would seek to use the relatively high-value therapies between the threshold points on Fig. 1 (B - C) and avoid the low-value therapies. There would be a 'sweet spot' with maximum outcome and a high value at point C.

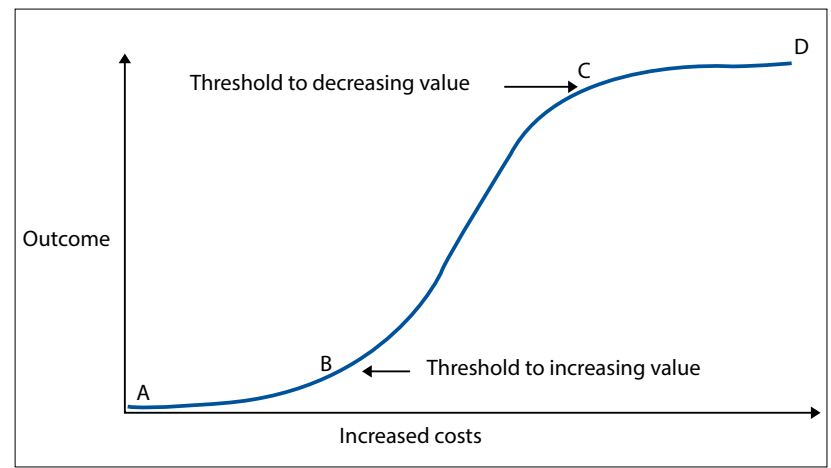

Fig. 1. A model for the change in outcome with increasing costs. (Value = outcome/costs.)

This model has been helpful in discussions by the author and others on the inclusion of different therapeutic interventions in treatment protocols, as it provided a framework for debate. Although the model is semiquantitative, agreement could readily be arrived at regarding on which part of the curve a proposed therapeutic intervention might fit.

The decision to include a therapeutic intervention in available treatment protocols will also need to include other factors, such as affordability. For example, the new biological therapies for melanoma $^{[2]}$ prolong survival, but are too expensive to use on a population-wide basis.

Funding in medicine is most often described from the perspective of medical schemes, government and industry. This needs to be complemented by the perspective of doctors who use their available resources to formulate cost-effective treatment protocols. The model may be helpful to doctors in joint undertakings with funders to provide quality care to patients and control costs.

\section{Raymond P Abratt}

Head of Clinical Governance, Independent Clinical Oncology Network, and Professor Emeritus, University of Cape Town

raymond.abratt@cancernet.co.za
1. Porter ME. What is value in health care? N Engl J Med 2010;363:2477-2481. [http://dx.doi.org/10.1056/ NEJMp1011024]

2. Robert C, Schachter J, Long GV, et al Pembrolizumab versus ipilimumab in advanced melanoma. N Engl J Med 2015;372:2521-2532. [http://dx.doi.org/10.1056/NEJMoa1503093]

S Afr Med J 2015;105(11):884. DOI:10.7196/SAMJ.2015.v105i11.9880 